\title{
Management of the engineering data for manufacturing
}

\author{
Gurcan Atakok* and Mufit Cun \\ Marmara University, Faculty of Technology, Department of Mechanical Engineering, Maltepe, 34854, Istanbul, Turkey. \\ *Corresponding Author: gatakok@marmara.edu.tr
}

$\begin{array}{ll}\text { Submitted } & : 26 / 08 / 2020 \\ \text { Revised } & : 24 / 06 / 2021 \\ \text { Accepted } & : 03 / 07 / 2021\end{array}$

\begin{abstract}
Most companies have adopted the use of computer integrated engineering software to cope with the increasing competition and pressure to produce better quality and at a faster rate, enhancing their systems on this basis. Models are designed on computers, designs are validated in the software, and tool paths for manufacturing are generated in different specific software. All engineering information is generated and stored in computers. This development enhances faster rate of works in companies; however, some problems such as the management of the data arise. All departments in a business work separately, but for the same purpose. It is required for each department to have access to related information. The existence of a system in which the information is checked and managed depending on their creation time, versions, and the department is of importance. In this article, a system that allows not only the mechanical design department, but also the manufacturing, storage, process planning, quality control, electrical design, purchasing departments, etc. to have access to the required information has been developed. In the absence of this kind of system, loss of time, scraps and loss of engineering time would be investigated. After starting up the system, its benefits are defined. Tracing the workflows for management purposes would be handled by the system. A 'Validation of Design' process is modelled for the company. The information can be accessible to only those responsible for the data, and not anyone else. This allows users to work in a better environment, hence, increases productivity.
\end{abstract}

Keywords: Concurrent engineering; Industry 4.0; Product data management; Product life cycle management.

\section{INTRODUCTION}

In the Era of Industry 4.0 and due to the current competitive market environment, there is a gradual increase in the expectations customers. Besides, new restrictions are being imposed by different authorities for environmental and security reasons. Sometimes, a revision must be applied to the existing product designs for the sake of variability in expectations. Product developers work in such a digitalized environment where all product-related information is stored and distributed (Scheidel et al., 2017). Although the product structures are information generated by the engineers, all the departments are required to have access to the information throughout the project's cycle. Therefore, there is a need for proper management of the product-related information. Product specifications and useful information are crucial factors for the success of businesses and for competitive advantage (Han et al., 2017; He et 
al., 2006; Papinniemi et al., 2014). In the concept of Industry 4.0, new products are manufactured consistently. In the meantime, 'smart' features are added to existing ones, thus, extending product variety. While we confront the smart drills in a manufacturing environment, smartwatches, smart toothbrushes and so on are being confronted in the commercial fields. Another challenging part of businesses is the development of information technologies. This requires collaborations and cooperation in the first phases of product development. Product development is important especially in the case of decreasing overall costs. About $60 \%$ to $80 \%$ of the overall costs are defined through the concept and pre-modelling design phases. When the problems are discovered in a later period, the cost would be higher. In other words, if the problem appears one phase later, it would cost ten times what it would have cost. Therefore, the development of design processes enhances the cost-saving potential (Haas et al., 2016; Ovtcharova, 2010).

\section{IMPORTANCE OF THE INFORMATION IN THE NEW PRODUCT DEVELOPMENT PROCESSES}

When the subject of a new product development conforms to the business targets, the new product development team may have to start the development processes over again. The relationship between the program and project managers cannot be estimated through purchasing and selling. More important than that, the product must be provided to the market in time and budget (Borasi \& Nagaich 2018). The complexity management is very important in the case of coordination and application of the product standardization scales in improving the market pricing. Thus, companies struggle with complexity and its costs from product variety. This requires analyses of the products and their components to eliminate product complexity (Schuh et al. 2018). Since new product development is a very important subject for businesses, specific factors are defined. Thus, many studies have been carried out to ensure the success of such factors (Montoya-Weiss \& Calantone, 1994). From the studies, the criteria for the success of the factors have been consolidated, simplified and generally, the specifications which include the Cross function working, support of top-level management, market planning and formulized processes are accepted. However, there exists only limited literature related to product development based on marketing perspectives and the engineeringbased sustainability cycle perspective (Gmelin \& Seuring). New product development can be described as a series of processes through which engineers conceptualize, design, and manufacture new products. In the last four decades, great efforts have been made to minimize the costs and design cycle time while preserving the competitive advantage. In the 1960s, products were designed by engineers without any structural process, hence, if the need for changes arose, it would call for product redesign. To meet up with the contemporary standard, a vast number of manufacturers have adopted the new system of product development. In Figure 1 the factors affecting the new product developments are represented. Production capacity, resources, company culture, customers, management and strategical positioning have a great role in this development. In the $1990 \mathrm{~s}$, only $40 \%$ of the industry had formed for new product development. This number increased tremendously to about $90 \%$ in the $2000 \mathrm{~s}$. Currently, there is a standard for new product development called the British Standard 7000; Guide to Managing Product Design (Nallusamy et al., 2015).

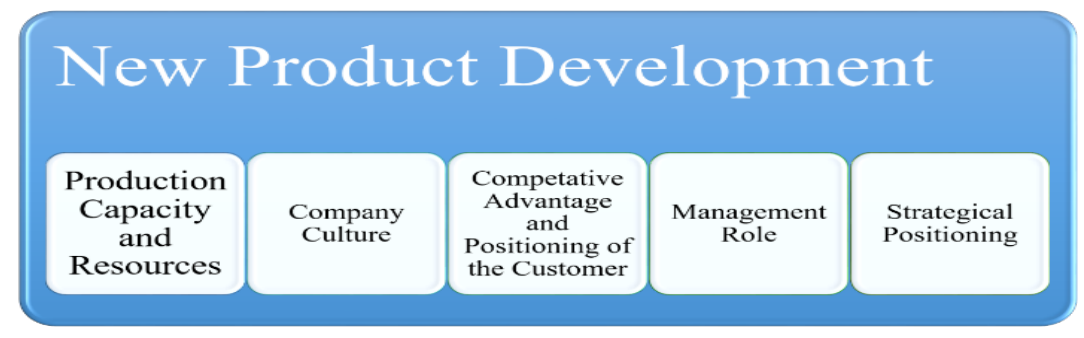

Figure 1. The Factors of the New Product Development. 
If the useful data are available in the design and engineering phases, there would be a decrease in the complexity and costs of the later phases of the product development cycle, especially in the manufacturing phase. Good data quality ensures product manufacturing, devoid of major revisions (Haas et al., 2016). Engineering outputs mainly contain the part specifications, cost of materials, technical drawings, user manuals, control lists and revision history. On the other hand, the project timeline and management's notes are cruel information for product developments (Han et al., 2017). During the new product development process, a huge amount of data is generated in the companies as the use of specific computer technologies increases gradually. This data is generated especially during design and manufacturing processes. This data can be sorted into two groups: the process and product data. Process data refers to the data related to the processes through which products are being manufactured. Engineering change orders, project authorizations, configurations of the product, workflows and information exchanges are considered as process data. Product data, on the other hand, can be classified into two groups: technical and nontechnical data. Nontechnical data involves all product costing, marketing information, sales orders, purchase orders, etc. Technical data contains the product design specifications, 3D models, technical drawings and assembly information, analysis reports, manufacturing Computer Numerical Control (CNC) codes and so on (Nallusamy et al., 2015; Staisch et al., 2012).

\section{PRODUCT DATA MANAGEMENT SYSTEMS}

Product Data Management (PDM) systems were introduced in the 1990s. It allows users and designers to obtain information from the Computer Integrated Manufacturing (CIM). In addition to its benefits such as concurrent engineering and allowing the user to access advanced manufacturing systems, it is also used to manage non-computeraided design (CAD) that may be generated during other phases of the production cycle. PDM allows users to have only the right information at the right time and in the right way; hence, it is an important tool for modern companies (Jin et al., 2014). It provides an environment in which the product information and the product development processes can be managed in a specific way. The data monitored usually includes the product's technical information, development and manufacturing information and material classes. A variety of expenses required to generate and sell the products can also be monitored using the PDM. PDM provides several services to store information on the process and product history and allows collaborations and data sharing within all users and to the person who generates the product such as management, engineering teams, sales department, customers and quality control department. Most of the PDM systems provide essential functionalities involving vault, document management, parts sorting, product structure and configuration management, workflow management and project management for new product development teams (Nallusamy et al., 2015). The contents used for explaining other data related to the products in PDM are represented in Figure 2. Not only the CAD Model, but also the analysis, drawings, CAM strategies, and quality control are very important. 


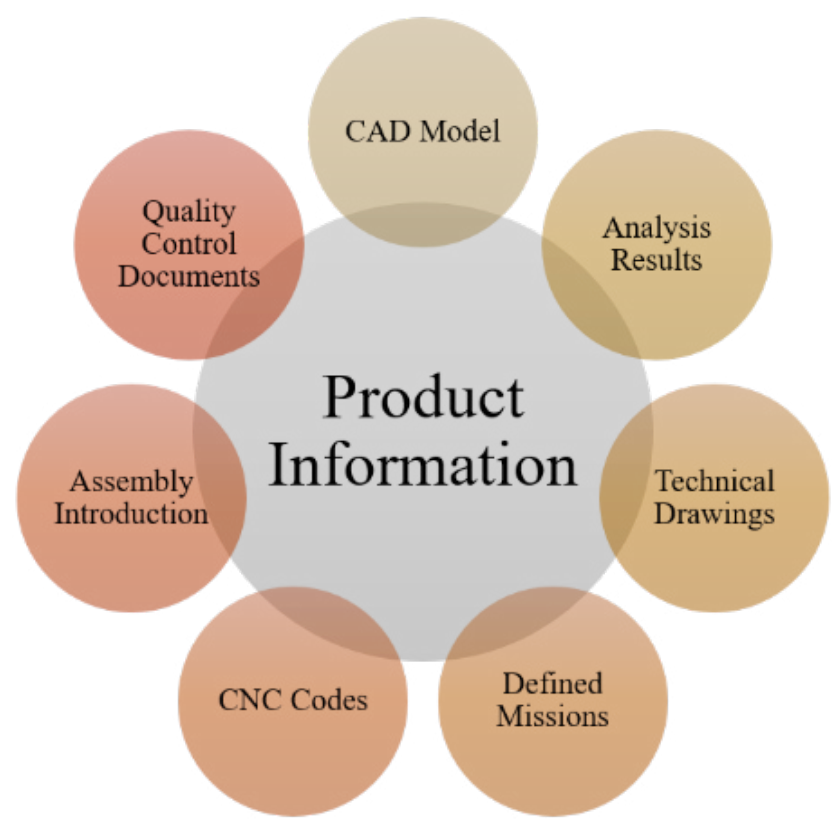

Figure 2. Data which create product data.

The system should involve a basic technique of registering information outputs in order to achieve the targets for having and using information through the project phases. Therefore, the structure of the system should be designed to obtain project schedule management, output acquisition process, and approval process integrated (Han et al., 2017). To create a proper flow, the fact that the system works by separated workflows should be considered. Another consideration to be taken while starting a new system or maintaining an existing one is whether the system will be adapted to the existing workflows or the workflows will be adapted to the system. For a successful start-up, all the organizations must be observed properly, and the processes should be adapted correctly (Kung et al.,2015).

\section{IMPORTANCE OF PDM SYSTEMS AND LITERATURE REVIEW}

Mesihovic et al. (2004) emphasized that the use of PDM systems for engineering processes and new product development processes must be divided into phases, especially for big projects as those that take a long time and the expensive ones. Philpotts (1996) stated that the existing data for product development increases with the enhancement of engineering software. He also stated that the importance of PDM systems is to generate, reuse and transfer in point of view. Huhtala et al. (2012), focused on the differences between PDM and the product lifecycle management (PLM) systems. PDM system is also analyzed. Merja and Harri (2017) studied sheet metal production, identifying several issues associated with the manufacturing processes of designed sheet metal products and their possible solution, as well as improvement in the product quality using PDM systems. Kropsu-Vehkapera et al. (2009) executed a project on the use of PDM in a high-tech company. Kumar \& Midha (2001) defined the quality function in order to use PDM systems for collaborative product development and also developed an approach in this context. The criteria to be investigated in order to choose the correct PDM system were also developed. Abdissa et al. (2018) investigated the PDM system usage in a textile factory and revealed some benefits of the system. Scheidel et al. (2018) applied a PDM project at a university environment to show the viability of PDM systems in small and medium-sized 
enterprises. Scheidel et al. (2017) compared six different PDM software and discovered some of the advantages and disadvantages of different approaches. In 2012, Staisch et al., through their studies, identified PDM systems as the base of PLM systems. Nallusamy et al. (2015) investigated the reasons PDM systems are needed during new product development processes and also defined the contributions of the systems. Haas et al. (2016) studied the importance of PDM systems in manufacturing processes. Ovtcharova (2010) investigated the assignment and position of PDM systems for the concept of virtual engineering. Gmelin \& Seuring (2014) emphasized that PDM systems are very important in the development processes of a sustainable new product. In Tao \& Qi (2019), the positions of PDM systems were defined in the context of information technologies based on smart manufacturing. Kim et al. (2001) investigated the standardizations of PDM systems and the PDM system to be chosen in the concept. Do (2014) explained the importance and necessity of PDM, not only for manufacturing but also for increasing service qualities. Jin et al. (2014) applied PDM system in an agricultural machine producer, investigating its benefits. Han et al. (2017) investigated PDM systems within the PLM in terms of generating and managing engineering information. Danjouet al. (2017) emphasized the steps employed in manufacturing, and the importance of PDM in the concept of CIM. In Do (2017), PDM systems are utilized in production using additive manufacturing technologies.

\section{CONCURRENT ENGINEERING}

Industries aim at manufacturing better and cheaper products, and yet, at a faster rate by employing concurrent engineering. Concurrent engineering is an approach that shortens the production time, increases product quality and decreases the operational and enhancement costs by implementing parallel workflows. It is enabled by finding all contributors, mainly the suppliers and customers, full integration of all the people from different specialties and conditions and enabling all the contributors to join most events as much as possible. Also, it helps the management reach a variable target related to the designing and manufacturing of complex products. In the past, most companies preferred consecutive workflows to parallel ones. This caused long delivery times and costly products. The low profit ended situation was due to a high volume of scrap, repetitive works and unproductive utilization of existing skills and technologies. Concurrent engineering is basically an integrated and simultaneous process in which productrelated information is shared among and clearly understood by all the engineers or users in a company. Consequently, it strives in highly heterogeneous environments where bidirectional data from below and above the organizational level are of importance in the observation of the data. Traditional paper-based data management and single computers cause disordered communication, loss of data and incorrect progress. Concurrency requires communication infrastructures for high tech industries such as information technologies, aviation, electronics and computer industries. PDM systems are identified as tools for enhancing concurrent engineering (Nallusamy et al., 2015; Staisch et al., 2012).

\section{COLLABORATIVE PRODUCT DEVELOPMENT}

Collaboration among departments and businesses enhances better decision making between several options and also allows the measuring of results to find out the best solution in the sustainable new product development process (Klassen \& Vachon, 2003). Eventually, even though collaboration is needed for the improvement of products, to shorten the production cycle time and to decrease the production costs, several companies fail to contribute to collaborations due to improper communication or lack of compatibility between the processes (Johnson, 2010; Barratt 2004). In a collaborative environment, tools, collaborative standards and structures, etc. need to be organized properly in order to not to interfere with the collaborations (Heuer, 2011). Product development projects are correlated with such applications that support developers and define milestones and as well, are restricted to specific cases. A collaboration is correlated with technologies and well-organized workflows, thus, there is a need to organize the contributor stakeholders around these requirements and related workflows (Johnson,2010; Cooper et al., 1997). 
Within this scope, exhaustive awareness of the roles of the team members in development teams is of importance to aid collaboration and the elimination of barriers. Barriers cause introversion, which interrupts new product development processes. To possess a sustainable new product development process and competitive advantage, companies need to get rid of such introversion and interiorize product or process-based approaches (Zhang, 2011; Chen \& Paulraj, 2004; Gmelin \& Seuring, 2014).

\section{THE REASONS FOR THE EMERGENCE OF PDM SYSTEMS}

The emergence of PDM systems was given rise to by the following situations, which are still being combated in manufacturing industries.

- Nowadays, related to the increasing usage of sophisticated CAD software in businesses, there exist two kinds of archives; the hardcopy and digitalized archives. Currently, some companies save up to $60 \%-70 \%$ of their data on papers. This is a traditional method of saving data and a worse means of storage due to the difficulty in the usage of the stored information. To manage hardcopies and digitalized archives and to enable the transition between two archives are among the greatest concerns of contemporary industries.

- The complexity of computer systems may be completely related to the volume of product data to ensure constructive decision makings. To appropriately access data located in different departments in a company, there may be need to integrate multiple computer systems. This is especially important as the use of common commercial software may result in the emergence of unmanageable computers and product information.

- All applications have their specific management functions. This causes differences in the definitions and structures of the parts. Different representations of the existing products are important in the case of various needs of different departments. For example, in the case of products in the product development processes, existing specifications required by this department prevent unnecessary changes and waste of time. This kind of representation is provided by different folder visualization features (Hoffman \& Joan-Arinyo, 1998; Nallusamy et al., 2015).

\section{UTILISED SOFTWARE MATERIALS}

Although there are several software devices in the industries, SolidWorks is chosen for this study due to its prevalent usage and ability to be integrated between electrical and mechanical designs. In addition to these, it is thought that having integrated analysis solutions may provide ease of engineering processes. Microsoft structured query language (SQL)was chosen as it is the most used SQL and it works collaboratively with SolidWorks. Office software are used for engineering processes such as transferring and investigating data. Although SolidWorks PDM supports all kind of data format, Microsoft Office was chosen in this study. To ensure full integration of this software, it is required that the correct versions be chosen.

\section{UTILISED PHYSICAL TOOLS}

To start the installation, the server is first installed. One or two server computers and clients' machines to receive data from servers are required. Engineering data are generated on the client machines and stored on the servers. To view these data without making changes, the screens are located close to the $\mathrm{CNC}$ machines. This helps in reducing ambiguity in manufacturing. 


\section{PREPARATION OF PDM SYSTEM IMPLEMENTATION}

The need to move to the new system of production emerged due to the obligation of engineers to work together for product development and should there be need to design similar products thereafter, they must start from scratch. This slows down not only the design processes but also other departments working collaboratively to sell products such as purchasing, marketing and technical support departments. Thus, the effected departments are listed to get involved in the system.

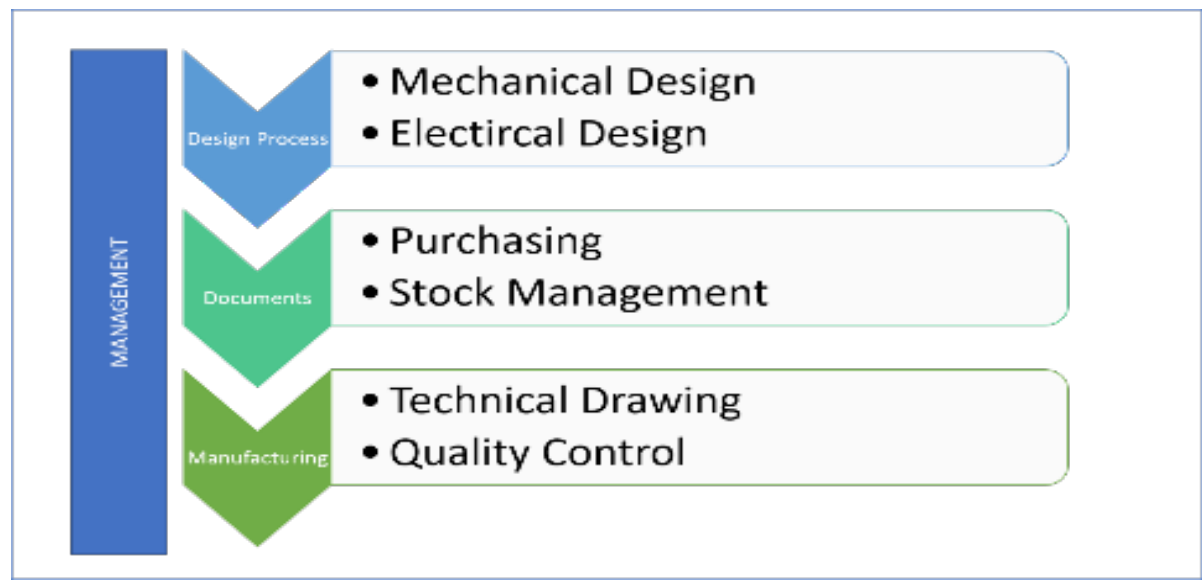

Figure 3. The Contributor of the PDM system.

The contributors to the system are represented in Figure 3. In this figure, since collaborations are obligations between them, electrical and mechanical design teams appear at the top of the list. The manufacturing department follows, as they need to follow up on the data, the progress of the manufacturing and assembling of the whole machine correctly. The quality control team can be considered at the same level as the manufacturing department as they use the same documents with the manufacturing team to ensure the products meet up with the specified standard. The purchasing and stock departments are also argued to be considered to provide proper parts at the correct time. To have control over all the processes and plan validation processes, the management is affected in each step.

\section{PDM FIELD APPLICATION}

The company already uses 3D CAD software. After the regulation of workflows, users and authentications, the existing projects are transferred to the PDM.

First, the employee's accounts and the workflows into which the designers are to transfer their projects are generated. Using these workflows, the system is designed for each user to work on their authenticated field.

After the integration of the PDM systems, collaboration is established to enable collaborative works by multiple users. Thus, multiple users can carry out their specific duties simultaneously on the same assembly model. Having a screen tells the version being used, who uses the version, etc., during the mechanical design process. This significantly minimizes the confusion that may arise. While working on this new environment, when the state of a product changes, as previously defined, the concerned users would be notified. For example, when a project is sent for approval, a 
notification would be sent to the user(s) who is in a position to approve it, when the design is approved, the concerned users would as well be notified. Therefore, the users would be continuously kept posted about each design and the situations. The electrical design and assembly team is integrated into the system. The teams make virtual assembly on software before the real execution in the field. In addition to this, for efficient cabling in the machine and collaborative work with the mechanical design team, the PDM environment should be employed. The positions of the components, as well as the cable lengths, become clearly known to both teams. In Figure 4, the electrical components and virtual assembly is represented. Hydraulic and pneumatic component assembly on the chassis and cabinet has been transferred to virtual assembly of electrical component and cabling.

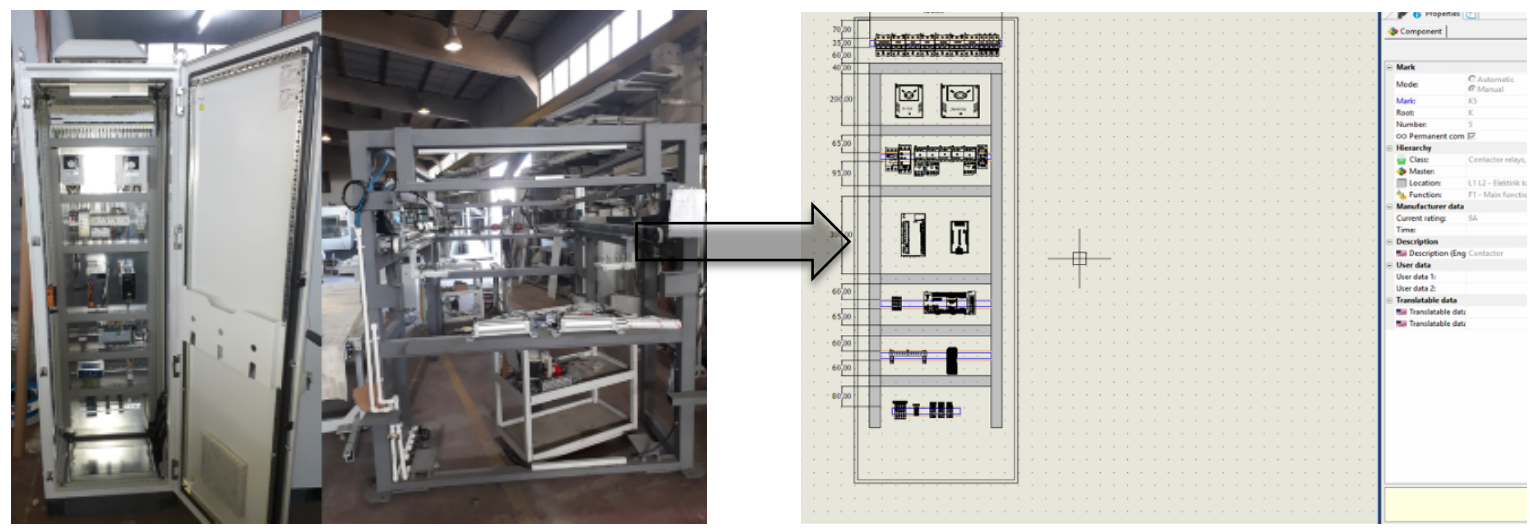

Figure 4. The electrical components and virtual assembly.

By generating all the technical drawings within this system, the required information can be shared with the CNC machines, as well as the conventional ones, to ensure the information about the part(s) to be manufactured is current and correct. For these machines, the required manufacturing time for each part is provided to the operator through PDM. Also, this information is sent to the MRP software generated by the company to help in management of future manufacturing plans based on the manufacturing times. Using these information, the company managers will be available to obtain comprehensive outputs such as the station at which the bottleneck would be located, the raw material to be purchased, the employee that works and how he/she works, the reasons for any mistake, should there be and the part to be manufactured at any station. Besides these, it is also possible to follow up on all the projects by observing the operations and the conditions of the parts. The mechanical and electrical design teams, as well as the management department, can access the same information at the same time through this integration. This ensures a more understandable product for all companies. In addition, assembly manuals are shared within the assembly team and the technical service team in PDM to improve productivity. These assembly product manuals are prepared in the PDM system so that if any product model is changed, the manuals would automatically be changed as well. All the system has become a structured mechanism without any data lose or missing the steps as shown in the Figure 5. 


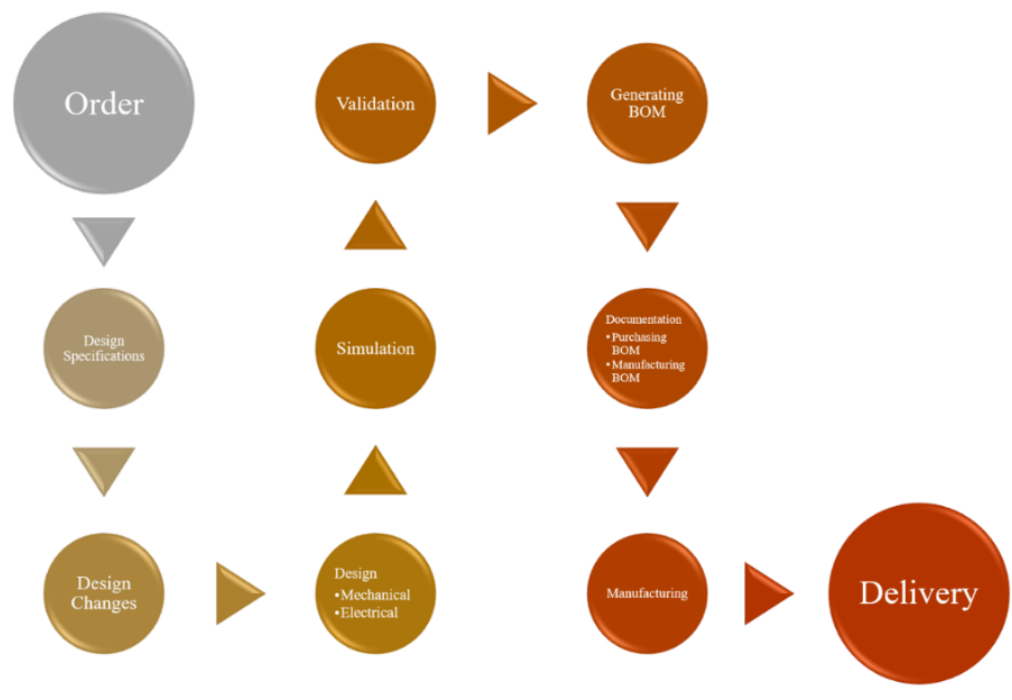

Figure 5. Overall Order to Delivery Representation of the Company.

\section{DISCUSSION AND CONCLUSION}

Since the company develops big projects, the first benefits are obtained in the mechanical design department by using a database approach. Consequently, the engineers who need to work on the same assembly can simultaneously access the same data. After the integration with the MRP systems and the manufacturing lines, the manufacturing team only utilizes the last revised version of the data, producing the required part(s) with accurate dimensions. The development processes for new products are faster since the existing data and models are being used. The reason for this is the decrease in the mistaken or repeated part numbers. As the result of working collaboratively, and with up to date data with no concerns, gradually scrap part manufacturing and decision-making time is decreased as depicted in Figure 6.

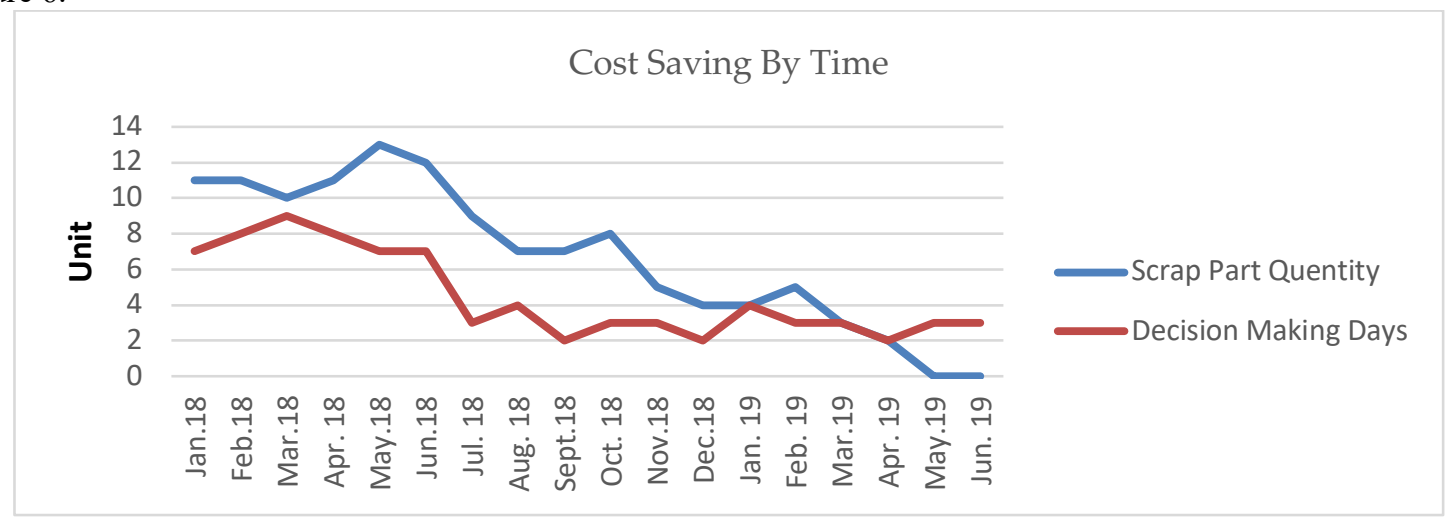

Figure 6. Alteration of invisible costs because of ineffectual working.

Not only the mechanical design team processes but also that of the contributor's decision making is hastened. The changes done in the mechanical design field can be observed from the electrical effects concurrently. In addition, 
the results can be investigated in terms of product and profitability. This system is highly recommended for such companies that continuously focuses on research and development. The required information for managing and control of the company is provided on forms. Below are questions answered and received to get information from this section.

- To edit or revise an existing design takes 1 work/week.

- As the data of the product is stored in different computers, to find the appropriate model takes $25 \mathrm{~min} / \mathrm{part}$.

- When any part is changed, to find the effected designs and to fix the unexpected behavior takes $20 \mathrm{~min} / \mathrm{part}$.

- In case there is no protocol to confirm that the right design is sent to the manufacturing team, an average of 11 parts are sent to manufacturing.

- To validate the design processes and components between the electrical and mechanical teams takes 3 days.

- To validate this information, the steps are checked with a chronometer.

After implementing the PDM system, the same topics were investigated for new sample projects. The results are listed below.

- To edit or revise an existing design takes 3 days/week.

- As the data for the product is stored in different computers, to find the correct model takes $2 \mathrm{~min} / \mathrm{part}$.

- When any part is changed, to find the effected designs and to fix the unexpected behavior takes $1 \mathrm{~min} /$ part.

- As a protocol is established to check whether the correct version of the part is sent to the manufacturing department, no wrongly manufactured part is observed.

- $\quad$ To validate this information, the steps are checked with a chronometer.

To understand result of two surveys Table 1 can be observed.

Table 1. Comparison of before/after PDM situations.

\begin{tabular}{|c|c|c|c|c|c|}
\hline & $\begin{array}{c}\text { Data } \\
\text { Revision }\end{array}$ & $\begin{array}{c}\text { Finding } \\
\text { Legacy Data }\end{array}$ & $\begin{array}{c}\text { Solving } \\
\text { Change Issues }\end{array}$ & $\begin{array}{c}\text { Wrong Part } \\
\text { due to } \\
\text { Validation }\end{array}$ & $\begin{array}{c}\text { Concurrent } \\
\text { Design Decisions } \\
\text { Making }\end{array}$ \\
\hline Before PDM & 7 days & $25 \mathrm{~min} /$ part & $20 \mathrm{~min} / \mathrm{part}$ & $11 \mathrm{part} /$ project & 3 days \\
\hline After PDM & 3 days & $2 \mathrm{~min} / \mathrm{part}$ & $1 \mathrm{~min} / \mathrm{part}$ & 0 part $/$ project & 0 days \\
\hline
\end{tabular}

Creating a PDM solved all the problems previously observed. For growing companies, their needs are expected to also grow, and non-avoidably differ daily. For developed new requirements or needs, better and improved product life management cycle solutions may be required in the future. With the help of this study, should there be a need for this kind of solution, the company and its infrastructure would be able to take necessary actions. With the help of this infrastructure, the company can now create the required data transform for several technological systems. This provides flexibility in the choice of possible solutions. Finally, the peak of this business would be to create all these systems in the cloud environment, independent of hardware. With this, in addition to all operational concerns, cyber security concerns resulting from digitalization would be compensated. The need for physical equipment will be reduced, thus, reducing the cost of infrastructure. 


\section{REFERENCES}

Abdissa, S., Worku, A., \& Shekar, C. (2018). Design and Development of Product Data Management (PDM) For Textile Company. J Textile Sci Eng, 8(370), 2.

Barratt, M. (2004). Understanding the meaning of collaboration in the supply chain. Supply Chain Management: an international journal, 9(1), 30-42.

Borasi, A., \& Nagaich, R. (2018). Product Development Process Using Tendering and Bidding.

Chen, I. J., \& Paulraj, A. (2004). Towards a theory of supply chain management: the constructs and measurements. Journal of operations management, 22(2), 119-150.

Cooper, M. C., Lambert, D. M., \& Pagh, J. D. (1997). Supply chain management: more than a new name for logistics. The international journal of logistics management, 8(1), 1-14.

Danjou, C., Le Duigou, J., \& Eynard, B. (2017). Closed-loop manufacturing process based on STEP-NC. International Journal on Interactive Design and Manufacturing (IJIDeM), 11(2), 233-245.

Do, N. (2014). Developing a product data model using the STEP PDM schema for integrating specifications of technical services. International Journal of Computer Integrated Manufacturing, 27(4), 382-399.

Do, N. (2017). Integration of design and manufacturing data to support personal manufacturing based on 3D printing services. The International Journal of Advanced Manufacturing Technology, 90(9-12), 3761-3773.

Gmelin, H., \& Seuring, S. (2014). Achieving sustainable new product development by integrating product lifecycle management capabilities. International Journal of Production Economics, 154, 166-177.

Haas, K., Schuck, H., Mücke, T., \& Ovtcharova, J. (2016). A holistic product lifecycle management approach to support design by machine data. Procedia CIRP, 50, 420-423.

Han, J., Lee, S. H., \& Kim, J. K. (2017). A process integrated engineering knowledge acquisition and management model for a project based manufacturing. International Journal of Precision Engineering and Manufacturing, 18(2), 175-185.

He, W., Ming, X. G., Ni, Q. F., Lu, W. F., \& Lee, B. H. (2006). A unified product structure management for enterprise business process integration throughout the product lifecycle. International Journal of Production Research, 44(09), 1757-1776.

Heuer, M. (2011). Ecosystem cross-sector collaboration: conceptualizing an adaptive approach to sustainability governance. Business Strategy and the Environment, 20(4), 211-221.

Hoffman, C. M., \& Joan-Arinyo, R. (1998). CAD and the product master model. Computer-Aided Design, 30(11), 905-918.

Huhtala, M., Lohtander, M., \& Varis, J. (2012) Confusing of terms PDM and PLM: examining issues from the PDM.

Jin, X. P., Zhang, T., \& Song, Z. H. (2014). A Windchill-Based PDM Application to Enhance Agricultural Machinery Chassis Design. In Applied Mechanics and Materials (Vol. 457, pp. 1653-1656). Trans Tech Publications.

Johnson, M. E. (2010). Product/Service Design Collaboration: Managing the Product Life Cycle. Wiley Encyclopedia of Operations Research and Management Science.

Kim, Y., Kang, S. H., Lee, S. H., \& Yoo, S. B. (2001). A distributed, open, intelligent product data management system. International Journal of Computer Integrated Manufacturing, 14(2), 224-235. 
Klassen, R. D., \& Vachon, S. (2003). Collaboration and evaluation in the supply chain: The impact on plant-level environmental investment. Production and Operations Management, 12(3), 336-352.

Kropsu-Vehkapera, H., Haapasalo, H., Harkonen, J., \& Silvola, R. (2009). Product data management practices in high-tech companies. Industrial Management \& Data Systems, 109(6), 758-774.

Kumar, R., \& Midha, P. S. (2001). A QFD based methodology for evaluating a company's PDM requirements for collaborative product development. Industrial Management \& Data Systems, 101(3), 126-132.

Kung, K. H., Ho, C. F., Hung, W. H., \& Wu, C. C. (2015). Organizational adaptation for using PLM systems: Group dynamism and management involvement. Industrial Marketing Management, 44, 83-97.

Merja, P., \& Harri, E. (2018). Challenges of manufacturability and product data management in bending. The International Journal of Advanced Manufacturing Technology, 99(9-12), 2137-2148.

Mesihovic, S., Malmqvist, J., \& Pikosz, P. (2004). Product data management system-based support for engineering project management. Journal of Engineering Design, 15(4), 389-403.

Montoya-Weiss, M. M., \& Calantone, R. (1994). Determinants of new product performance: A review and metaanalysis. Journal of product innovation management, 11(5), 397-417.

Nallusamy, S., Balakannan, K., Suganthini Rekha, R., \& Balasubramanian, K. (2015). A Review on Valuable Trends of Product Data Management (PDM) Occupied in New Product Development (NPD). In Applied Mechanics and Materials (Vol. 786, pp. 262-268). Trans Tech Publications.

Ovtcharova, J. G. (2010). Virtual engineering: principles, methods and applications. In DS 60: Proceedings of Design 2010, the 11th International Design Conference, Dubrovnik, Croatia.

Papinniemi, J., Hannola, L., \& Maletz, M. (2014). Challenges in integrating requirements management with PLM. International Journal of Production Research, 52(15), 4412-4423.

Philpotts, M. (1996). An introduction to the concepts, benefits and terminology of product data management. Industrial Management \& Data Systems, 96(4), 11-17.

Scheidel, W., Mozgova, I., \& Lachmayer, R. (2017). Product Data Management in the Context of Industry 4.0. In Engineering for a Changing World: Proceedings; 59th IWK, Ilmenau Scientific Colloquium, Technische Universität Ilmenau, September 11-15, 2017 (Vol. 59, No. 4.2. 05).

Scheidel, W., Mozgova, I., \& Lachmayer, R. (2018). Teaching Industry 4.0-Product Data Management for Small and Medium-Sized Enterprises. In DS 93: Proceedings of the 20th International Conference on Engineering and Product Design Education (E\&PDE 2018), Dyson School of Engineering, Imperial College, London. 6th-7th September 2018 (pp. 151-156).

Schuh, G., Dölle, C., Schmitz, S., Koch, J., Höding, M., \& Menges, A. (2018). Data-Based Determination of the Product-Oriented Complexity Degree. Procedia CIRP, 70, 144-149.

Staisch, A., Peters, G., Stueckl, T., \& Seruga, J. (2012). Current trends in product lifecycle management.

Tao, F., \& Qi, Q. (2017). New IT driven service-oriented smart manufacturing: framework and characteristics. IEEE Transactions on Systems, Man, and Cybernetics: Systems, 49(1), 81-91.

Zhang, D. Z. (2011). Towards theory building in agile manufacturing strategies - Case studies of an agility taxonomy. International Journal of Production Economics, 131(1), 303-312. 